Infusionsther Transfusionsmed 1997;24:1-2

\title{
Impressum, Vol. 24, No. 1, 1997
}

\section{Band 24, Heft 1,1997}

Offizielles Organ der Deutschen Gesellschaft für Transfusionsmedizin und Immunhämatologie inúusionsiherapie Transfusionsmedizin

Interdisziplinäre Zeitschrift für Infusions- und Hämotherapie, klinische Ernährung und

Intensivmedizin

Gründungsherausgeber

H. Reissigl, Innsbruck

Herausgeber

J. Eckart, Augsburg V. Kretschmer, Marburg W. Mempel, München K. Meßmer, München K.

Peter, München W. Stangel, Hannover K.-H. Usadel, Frankfurt

Schrîftleiter

H. Forst, Augsburg B. Zwißler, München

Wissenschaftlicher Beirat

M. Adolph, Augsburg

F.W. Ahnefeld, Ulm

C. Baldamus, Köln

H. Bardenheuer, Heidelberg

J.-F. Baron, Paris

W. Behrendt, Aachen

H. Borberg, Köln

U. B. Bruckner, Ulm

R. Eckstein, Erlangen

A. Encke, Frankfurt

U. Finsterer, München

L. Frey, München

Y. Fujita, Okayama

L. Gattinoni, Mailand

M. Georgieff, Ulm
A. Grünert, Ulm
H. J. Gurland, Neuried
P. Hanfland, Bonn
M. Heberer, Basel
J. P. Isbister, Sydney
K. Jauch, Regensburg
W.-P Kloevekorn, Bad Nauheim
H.-J. Kolb, München
W. Kox, Berlin
H. G. Kress, Wren
B. Kubanek, Ulm 


\author{
P. Kühnl, Hamburg \\ H. Laubenthal, Bochum \\ N. Mutz, Innsbruck \\ U E. Nydegger, Bern \\ W. Oettinger, Trier \\ Th. Prien, Münster \\ D. Roelcke, Heidelberg \\ R. Roos, München \\ W. Schleinzer, Hamburg \\ D. Schönitzer, Innsbruck \\ H.-G Sieberth, Aachen \\ U Suchner, München \\ L. Sunder-Plassmann, Ulm \\ E. Wenzel, Homburg/Saar \\ K. Werdan, Halle/Saale \\ K. Widhalm, Wien \\ G. Wolfram, München R. Zander, Mainz
}

\title{
KÄRGEIl
}

Bibliographischer Hinweis:

Inhaltsverzeichnisse dieser Zeitschrift erscheinen regelmäßig in current contents ${ }^{\circledR}$ sowie in anderen bibliographischen Diensten.

S. Karger $\cdot$ Medical and Scientific Publishers $\cdot$ Basel $\square$ Freiburg $\cdot$ Paris $\square$ London $\cdot$ New York New Delhi · Bangkok — Singapore · Tokyo — Sydney

Die Zeitschrift erscheint zweimonatlich; pro Jahr erscheint 1 Band zu je 6 Heften.

DGTLMitglieder erhalten die Zeitschrift im Rahmen ihrer Mitglíedschaft. Bezugspreis für Jahrgang24, 1997, DEM 182-/CHF 142,-, einschließlich MwSt., zuzügПch Postgebühren. Der Abonnementpreis ist im voraus zahlbar. Das Abonnement der Zeitschrift läuft weiter, wenn es nicht spätestens 4 Wochen vor Abschluß eines Bandes abbestellt wird. Abonnementbestellungen können bei jeder Buchhandlung oder direkt beim Verlag aufgegeben werden:

Bundesrepublik Deutschland: S. Karger GmbH Lörracher Str. 16 a D-79115 Freiburg Telefon (0761) 452070 Telefax (0761) 4520714 Postgiro München 61440-808

Übrige Lander: S. Karger AG Allschwilerstr. 10 Postfach CH-4009 Basel Telefon (061) 3061111 Telefax (061) 3061234 E-Mail Karger@Karger.ch

Anzeigen: S. Karger

Verlag für Medizin und Naturwissenschaften $\mathrm{GmbH}$

Lörracher Str. 16a, D-79115 Freiburg

Telefon (0761) 452070

Gültig ist die Preisliste Nr. 13 vom 1.Januar 1997.

Für den Inhalt außerhalb des redaktionellen Teiles (insbesondere Anzeigen,

Industrieinformationen. Presse-zitate und Kongreßinformationen) übernehmen Schrift-leitung,

Beirat und Verlag keine Gewähr. Eine Markenbezeichnung kann warenzeichenrechtlich

geschützt sein, auch wenn bei ihrer Verwendung in dieser Zeitschrift das Zeichen ${ }^{\circledR}$ oder ein anderer Hinweis auf etwa bestehende Schutzrechte fehlen sollte. Für Satz-fehler, insbesondere bei Dosierungsangaben, wird keine Gewähr übernommen. 
Die Zeitschrift sowie alle in ihr enthaltenen einzelnen Beiträge und Abbildungen sind urheberrechtlich geschützt. Jede Verwertung, die nicht ausdrücklich vom Urheberrechtsgesetz zugelassen ist, bedarf der vorherigen Zustimmung des Verlags. Das gilt insbesondere für Vervielfâlttigungen, Bearbeitungen, Übersetzungen, Mikro-verfilmungen und die Einspeicherung und Verarbeitung in elektronischen Systemen. Fotokopien dürfen nur für den persönlichen Gebrauch als Einzelkopien hergestellt werden. Jede im Bereich eines gewerblichen Unternehmens zulässig hergestellte oder benutzte Kopie dient gewerblichen Zwecken gem. § 54(2) UrhG und verpflich-tet zur Gebührenzahlung an die Verwertungsgesellschaft WORT, Abt. VG Wissenschaft. Goethestraße 49, D-80336 München

C Copyright 1997 by S. Karger

Verlag für Medizin und Naturwissenschaften. GmbH

Lörracher Str. 16 a, D-79115 Freiburg

Verlagsleitung und presserechtlich verantwortlich: Sibylle Hopf Assistenz: Susanne Meister Herstellung: Georg Brunner Anzeigenverwaltung: Christiane Opilz

Satz und Druck: Walter Biering GmbH Grafischer Betrieb Freisinger Landstraße 21 D-80939 München

W 